\title{
QUALIDADE DE VIDA E SOBRECARGA DE CUIDADORES DE CRIANÇAS COM PARALISIA CEREBRAL
}

\section{Quality of life and burden in caregivers of children with cerebral palsy Calidad de vida y sobrecarga de cuidadores de niños con parálisis cerebral}

\author{
Jandara de Moura Souza (Lattes) \\ Universidade Federal de Ciências da Saúde de Porto Alegre - UFCSPA - Porto Alegre (RS) - Brasil \\ Fabiana Rita Camara Machado (Lattes) \\ Hospital de Clínicas de Porto Alegre - HCPA - Porto Alegre (RS) - Brasil \\ Priscilla Pereira Antunes (Lattes) \\ Universidade Federal de Ciências da Saúde de Porto Alegre - UFCSPA - Porto Alegre (RS) - Brasil
}

Antônio Cardoso dos Santos (Lattes)

Universidade Federal do Rio Grande do Sul - UFRGS - Porto Alegre (RS) - Brasil

Daniela Centenaro Levandowski (OrcID)

Universidade Federal de Ciências da Saúde de Porto Alegre - UFCSPA - Porto Alegre (RS) - Brasil

Alcyr Alves de Oliveira Junior (OrcID)

Universidade Federal de Ciências da Saúde de Porto Alegre - UFCSPA - Porto Alegre (RS) - Brasil

\section{RESUMO}

Objetivo: Analisar a relação entre qualidade de vida (QV) e sobrecarga dos cuidadores relacionados ao grau de comprometimento neuromotor de crianças com paralisia cerebral (PC). Métodos: Estudo transversal realizado em 2014, com 38 cuidadores de crianças com paralisia cerebral atendidas pelo serviço de Fisiatria e Reabilitação do Hospital de Clínicas de Porto Alegre, Rio Grande do Sul, Brasil. Utilizaram-se os instrumentos World Health Organization Quality of Life Questionaire (WHOQOL-BREF), Beck Depression Inventory (BDI), Beck Anxiety Inventory (BAI), Inventário de Ansiedade Traço-Estado (IDATE) e Zarit Caregiver Burden Interview (ZCBI). Avaliou-se o grau de desenvolvimento motor das crianças pelo Gross Motor Function Classification System (GMFCS). Avaliaram-se as associações (coeficiente de Pearson) entre as variáveis descritas por média e desvio padrão, ou mediana, e amplitude interquartílica. Resultados: Encontraram-se 20 cuidadores (52,6\%) com sobrecarga moderada. Encontraram-se associações no domínio social da WHOQOL-BREF em relação ao desenvolvimento motor das crianças ( $\mathrm{rs}=0,355$; $p=0,029$ ) e associação positiva entre a ZCBI e os escores do IDATE ( $r s=0,540 ; p<0,001)$. Os resultados sugerem que, quanto maiores os níveis de ansiedade, de depressão e de sobrecarga dos cuidadores, menores serão os escores de QV dos cuidadores. Não se observaram associações entre os escores de QV e o grau de prejuízo motor da criança, e os escores de depressão e a ansiedade do cuidador. Conclusão: Há interações entre QV e demais aspectos emocionais dos cuidadores, entretanto não se constatou relações entre a QV dos cuidadores e o nível de desenvolvimento motor de crianças.

Descritores: Paralisia Cerebral; Qualidade de Vida; Depressão; Ansiedade; Cuidadores.

\section{ABSTRACT}

Objective: To analyze the association of Quality of Life (QoL) and caregiving burden with the degree of neuromotor impairment in children with cerebral palsy (CP). Methods: Cross-sectional study carried out in 2014 with 38 caregivers of children with cerebral palsy attending the Physiatry and Rehabilitation Clinic of the Hospital de Clínicas of Porto Alegre, Rio Grande do Sul, Brazil. The World Health Organization Quality of Life Questionnaire (WHOQOL - BREF), the Beck Depression Inventory (BDI), the Beck Anxiety Inventory (BAI), the State-Trait Anxiety Inventory (STAI), and the Zarit Burden Interview (ZBI) were used. The degree of children's motor development was assessed using the Gross Motor Function Classification System (GMFCS). We checked for associations (Pearson's coefficient) between variables and they were described as mean and standard deviation, or median, and interquartile range. Results: There were 20 caregivers (52.6\%) with a moderate burden. The domain Social Relationships, of the WHOQOL - BREF, was associated with children's motor development ( $r s=0.355 ; p=0.029)$, and there was a positive association between $Z B I$ and STAI scores ( $r s=0.540 ; p<0.001)$. The results suggest that the higher the degree of anxiety, depression and 
burden in caregivers, the lower the QoL scores in caregivers. The QoL scores were no associated with the child's degree of motor impairment or caregivers' depression and anxiety scores. Conclusion: There were interactions between caregivers' QoL and other emotional aspects, but there were no associations between caregivers' QoL and degree of motor development.

Descriptors: Cerebral Palsy; Quality of Life; Depression; Anxiety; Caregivers.

\section{RESUMEN}

Objetivo: Analizar la relación entre la calidad de vida (CV) y la sobrecarga de los cuidadores relacionados al grado de comprometimiento neuromotor de niños con parálisis cerebral (PC). Métodos: Estudio transversal realizado en 2014 con 38 cuidadores de niños con parálisis cerebral asistidos por el servicio de Fisiatría y Rehabilitación del Hospital de las Clínicas de Porto Alegre, Rio Grande do Sul, Brasil. Se utilizaron los instrumentos World Health Organization Quality of Life Questionaire (WHOQOL - BREF), el Beck Depression Inventory (BDI), el Beck Anxiety Inventory (BAI), el Inventario de Ansiedad Trazo-Estado (IDATE) y el Zarit Caregiver Burden Interview (ZCBI). Se evaluó el grado de desarrollo motor de los niños con el Gross Motor Function Classification System (GMFCS). Se evaluaron las asociaciones (coeficiente de Pearson) entre las variables descritas por la media y la desviación típica o la mediana, y la amplitud interquartílica. Resultados: Se han encontrado 20 cuidadores $(52,6 \%)$ con sobrecarga moderada. Se han encontrado asociaciones en el dominio social del WHOQOL-BREF respecto el desarrollo motor de los niños ( $r s=0,355 ; p=0,029)$ y asociación positiva entre la ZCBI y las puntuaciones del IDATE ( $r S=0,540$; $p<0,001)$. Los resultados sugieren que a mayores niveles de ansiedad, depresión y sobrecarga de los cuidadores menores son sus puntuaciones para la CV. No se ha observado asociaciones entre las puntuaciones de la CV y el grado de perjuicio motor del niño y las puntuaciones de depresión y ansiedad del cuidador. Conclusión: Hay interacciones entre la CV y los demás aspectos emocionales de los cuidadores, sin embargo, no se ha constatado relaciones entre la CV de los cuidadores y el nivel de desarrollo motor de los niños.

Descriptores: Parálisis Cerebral; Calidad de Vida; Trastornos de Adaptación; Ansiedad; Cuidadores.

\section{INTRODUÇÃO}

As políticas de promoção da saúde têm papel fundamental na redução da prevalência de casos de paralisia cerebral (PC) em países em desenvolvimento, onde a incidência é maior que em países desenvolvidos ${ }^{(1-3)}$. A promoção da saúde de cuidadores de crianças com PC relaciona-se fortemente com a qualidade de vida (QV) e com os níveis de sobrecarga dos cuidadores ${ }^{(1-3)}$. Muitas vezes, esses indivíduos declaram que se sentem sobrecarregados em suas atividades, gerando estresse, dificuldades de relações interpessoais e, até mesmo, causando adoecimento, assim necessitando de auxílio para a saúde física e mental ${ }^{(4,5)}$.

A PC é caracterizada por alteração dos movimentos controlados ou posturais, sendo secundária à lesão, ao dano ou à disfunção do sistema nervoso central, não sendo reconhecida como resultado de uma doença cerebral progressiva ou degenerativa ${ }^{(4)}$. A PC é uma condição patológica resultante de evento perinatal, tendo como principal causa de morbidade e mortalidade o nascimento prematuro antes da trigésima sétima semana de gestação ${ }^{(5,6)}$.

A principal alteração presente nas crianças com PC é o comprometimento motor, que afeta a biomecânica corporal, interferindo na vida diária. Também pode apresentar distúrbios cognitivos, sensoriais, visuais e auditivos que, somados às alterações motoras e às restrições de tarefas e de ambiente, repercutirão de diferentes formas no seu desempenho funcional( ${ }^{(7)}$. De fato, a heterogeneidade do quadro clínico apresentado por crianças com PC dificulta a classificação do comprometimento da disfunção motora, sendo um desafio para as equipes multiprofissionais envolvidas na reabilitação trabalhar com medidas baseadas no seu desempenho funcional(8,9).

A PC pode ser classificada por dois critérios: pelo tipo de disfunção motora, ou seja, o quadro clínico resultante, que inclui os tipos extrapiramidal ou discinético (atetóide, coréico e distônico), atáxico, misto e espástico; e pela topografia dos prejuízos, isto é, a localização do corpo afetado, que inclui tetraplegia ou quadriplegia, monoplegia, paraplegia ou diplegia e hemiplegia. Na PC, a forma espástica é a mais frequente estando presente em $88 \%$ dos $\operatorname{casos}^{(10,11)}$.

A prevalência da PC é de 2 a 3 por 1.000 nascidos vivos, sendo a causa mais comum de deficiência física grave em crianças. O diagnóstico de $\mathrm{PC}$, usualmente, envolve o retardo ou o atraso no desenvolvimento motor, a persistência de reflexos primitivos, a presença de reflexos anormais e o fracasso do desenvolvimento dos reflexos protetores $^{(12,13)}$.

As consequências da PC são associadas pelos familiares a sentimentos de tristeza, de fragilidade e de dependência ${ }^{(14,15)}$. O cuidador de uma criança com PC vivencia diversas situações na família no que tange ao 
exercício dos papéis familiares, de caráter financeiro e também sentimentos de desamparo, de perda de controle, de exclusão e de sobrecarga, os quais podem trazer a ansiedade e a depressão como respostas às dificuldades físicas e emocionais ${ }^{(16-19)}$. Essa situação pode comprometer a QV desses cuidadores ${ }^{(20,21)}$.

A Organização Mundial de Saúde (OMS) define QV como a percepção do indivíduo sobre a sua condição de vida no contexto da cultura e do sistema de valores nos quais vive e em relação aos seus objetivos, expectativas, padrões e preocupações ${ }^{(22)}$. Corresponde, também, ao grau de satisfação encontrado na vida familiar, sentimental, social, ambiental e na própria existência, sendo a expressão de muitos significados que abrangem e refletem conhecimentos, experiências e valores na vida do indivíduo ${ }^{(16,23,24)}$. Entende-se que esses aspectos são importantes nas ações de promoção, de prevenção, de tratamento e de reabilitação em saúde ${ }^{(24-27)}$. Assim, a QV e o nível de sobrecarga imposto aos cuidadores de crianças com PC em reabilitação demandam intenso envolvimento dos responsáveis.

Dessa forma, o presente estudo objetivou analisar a relação entre qualidade de vida e sobrecarga dos cuidadores relacionados ao grau de comprometimento neuromotor de crianças com paralisia cerebral.

\section{MÉTODOS}

Trata-se de um estudo transversal realizado com cuidadores de crianças com paralisia cerebral em Porto Alegre, Rio Grande do Sul, Brasil, em 2014.

A amostra por conveniência contou com 38 cuidadores de 38 crianças com diagnóstico de PC, com idades entre zero e 11 anos, de ambos os sexos, que estavam em atendimento no Serviço de Fisiatria e Reabilitação do Hospital de Clínicas de Porto Alegre (HCPA), classificadas em todos os níveis da escala Gross Motor Function Classification System (GMFCS).

Utilizaram-se os seguintes instrumentos validados para a população brasileira: World Health Organization Quality of Life - Short Version (WHOQOL-BREF), Beck Anxiety Inventory (BAI), Beck Depression Inventory (BDI), Trait Anxiety Inventory-State (STAI), Inventário de Ansiedade Traço-Estado (IDATE), Zarit Caregiver Burden Interview (ZCBI) e Gross Motor Function Classification System (GMFCS).

Aplicou-se, primeiramente, o WHOQOL-BREF, um instrumento que consiste em 26 perguntas referentes a quatro domínios: físico, psicológico, social e meio ambiente. A pontuação total e de cada domínio varia entre 0 e 100, em que, quanto maior o escore, melhor a $\mathrm{QV}^{(22)}$. No Brasil, o instrumento validado demonstrou uma consistência interna de $\alpha=0,9054^{(22)}$. Todavia não há, até o momento, dados normativos populacionais dos escores do WHOQOL, escala que originou o WHOQOL-BREF(22).

Em seguida, aplicou-se o BAI e o BDI. O primeiro tem o objetivo de avaliar a intensidade de sintomas ansiosos, sendo constituído por 21 afirmações descritivas de sintomas de ansiedade, avaliados numa escala de 4 pontos, refletindo os níveis de gravidade crescente de cada sintoma (leve, moderada e severa). Já o segundo avalia a intensidade dos sintomas depressivos. Trata-se de um inventário de autorrelato constituído por 21 itens, cada um com quatro alternativas, subentendendo graus crescentes de gravidade da intensidade dos sintomas (leve, moderada e severa). O escore total dos inventários resulta da soma dos itens individuais ${ }^{(28)}$. Os inventários Beck são amplamente usados em estudos clínicos e pré-clínicos, com populações diversificadas, sendo validados para uso no Brasil e demonstrando alta consistência interna, de $\alpha=91^{(29)}$.

Utilizou-se o Inventário de Ansiedade Traço-Estado (IDATE) para avaliar a ansiedade com características mais permanentes, inerentes ao sujeito (ansiedade-traço) e às reações do sujeito à situação atual (ansiedade-estado) ${ }^{(30)}$. Esse instrumento é dividido em duas partes: a primeira, correspondente ao momento da avaliação, é composta por 20 alternativas; a segunda apresenta 20 alternativas que retratam como o sujeito se apresenta na maior parte do tempo ${ }^{(31)}$. Esse teste demonstrou consistência interna de $\alpha=0,89^{(30)}$.

Para avaliar a sobrecarga do cuidador, utilizou-se o $Z \mathrm{ZBI}^{(31)}$, um inventário composto por 22 itens e que avalia o impacto das atividades de cuidar sobre a vida social e financeira, as relações interpessoais e o bem-estar físico e emocional do cuidador. As respostas devem ser dadas seguindo uma escala de cinco pontos, que descrevem como cada afirmação afeta a pessoa. Quanto maior a pontuação obtida, maior é o peso do cuidar. Esse teste demonstrou consistência interna de $\alpha=0,80^{(32)}$.

Ao final, avaliaram-se as crianças de acordo com a escala GMFCS, a fim de determinar o nível de desempenho habitual e as limitações da função motora grossa global desses pacientes. A GMFCS para PC é baseada no movimento iniciado voluntariamente, com ênfase no sentar, transferências e mobilidade, e sua classificação é baseada nos seguintes níveis: nível I - anda sem limitações; nível II - anda com limitações; nível III - anda utilizando um 
dispositivo manual de mobilidade; nível IV - auto-mobilidade com limitações, pode utilizar mobilidade motorizada; nível $\mathrm{V}$ - transportado em uma cadeira de rodas manual ${ }^{(33,34)}$.

Para analisar estatisticamente os dados coletados, descreveram-se as variáveis quantitativas por média e desvio padrão, ou mediana, e amplitude interquartílica, dependendo da distribuição da variável. Já as variáveis categóricas encontram-se descritas por frequências absolutas e relativas. Para comparar médias entre as categorias do estado civil (com ou sem companheiro), aplicou-se o teste t de Student. Avaliaram-se as associações entre as variáveis pelos coeficientes de correlação de Pearson (distribuição simétrica) ou Spearman (distribuição assimétrica). Para controle de fatores confundidores, aplicou-se análise de regressão linear multivariada. Utilizou-se o teste de regressão linear múltipla para avaliar as seguintes variáveis: idade, estado civil, ansiedade-traço, ansiedade-estado, sintomas ansiosos, sintomas depressivos, sobrecarga e nível de desenvolvimento motor da criança. Para avaliar o efeito de cada fator em relação aos escores de QV, calculou-se o coeficiente angular (b) em conjunto com o intervalo de $95 \%$ de confiança. Utilizou-se o coeficiente padronizado $(\beta)$ para comparar o efeito entre os fatores, e o método de extração Backward para a determinação do modelo final. Adotou-se como nível de significância $5 \%(p \leq 0,05)$, realizando-se as análises no programa SPSS, versão 21.0.

O presente estudo obteve aprovação do Comitê de Ética em Pesquisa da Universidade Federal de Ciências da Saúde de Porto Alegre, sob o Parecer $n^{\circ}$ 602.177-0. Todos os participantes assinaram o Termo de Consentimento Livre e Esclarecido.

\section{RESULTADOS}

$\mathrm{Na}$ amostra de 38 cuidadores, encontrou-se a média de idade de 39,8 anos (desvio padrão $=8,2$ ). Em relação ao sexo, 35 participantes $(92,1 \%)$ eram do sexo feminino e apenas $3(7,9 \%)$, do sexo masculino. Referente ao estado civil, $26(68,4 \%)$ eram casados, enquanto $10(26,3 \%)$ cuidadores eram solteiros e outros $2(5,3 \%)$ eram divorciados, conforme demonstra a Tabela I.

Tabela I - Caracterização da amostra de cuidadores. Porto Alegre, Rio Grande do Sul, 2014.

\begin{tabular}{lc}
\hline Variáveis & $\mathbf{n = 3 8}$ \\
\hline Idade (anos) média \pm DP & $39,8 \pm 8,2$ \\
Sexo & $\mathbf{n}(\%)$ \\
Masculino & $3(7,9)$ \\
Feminino & $35(92,1)$ \\
Estado civil & $\mathbf{n}(\%)$ \\
Solteiro & $10(26,3)$ \\
Casado & $26(68,4)$ \\
Divorciado & $2(5,3)$ \\
\hline
\end{tabular}

DP: desvio padrão; n(\%): número em porcentagem; n: número

Encontrou-se a seguinte distribuição do nível de PC das crianças: 19 crianças $(50 \%)$ atingiram nível II na GMFCS; 11 crianças $(28,9 \%)$ atingiram nível III; 4 crianças (10,5\%), o nível V; e outras 2 crianças $(5,3 \%)$ apresentaram nível I e nível IV, conforme a Tabela II.

Tabela II - Avaliação do nível de desenvolvimento motor das crianças através da escala Gross Motor Function Classification System (GMFCS). Porto Alegre, Rio Grande do Sul, 2014.

\begin{tabular}{lcc}
\hline Nível de desenvolvimento motor & $\mathbf{n}$ & $\%$ \\
\hline I - Anda sem limitações & 2 & 5,3 \\
II - Anda com limitações & 19 & 50,0 \\
III - Anda utilizando um dispositivo manual de mobilidade & 11 & 28,9 \\
IV - Automobilidade com limitações; pode utilizar mobilidade motorizada & 2 & 5,3 \\
V - Transportado em uma cadeira de rodas manual & 4 & 10,5 \\
\hline
\end{tabular}

n: número; \%: porcentagem

Em relação à intensidade dos sintomas de ansiedade avaliada pelo BAI: 18 cuidadores $(47,4 \%)$ apresentaram classificação mínima para ansiedade; $13(34,2 \%)$ apresentaram classificação moderada; 6 (15,8\%), classificação 
leve; e apenas 1 (2,6\%), classificação grave. Quanto à intensidade dos sintomas depressivos, os resultados do BDI apontaram que: 20 cuidadores $(52,65 \%)$ apresentaram classificação mínima; 10 (26,3\%), classificação moderada; $6(15,8 \%)$, classificação leve; e apenas 2 cuidadores (5,3\%), intensidade grave.

Referente à classificação de ansiedade traço-estado, medida pelo IDATE, considerando-se a ansiedade-traço, 22 cuidadores $(57,9 \%)$ tenderam à ansiedade, enquanto $12(31,6 \%)$ apresentaram nível normal de ansiedade, e outros $4(10,5 \%)$ tenderam à depressão. Já em relação à classificação da ansiedade-estado, 28 cuidadores $(73,7 \%)$ tenderam à ansiedade, $7(18,4 \%)$ apresentaram nível normal de ansiedade, e outros $3(7,9 \%)$ tenderam à depressão. Não houve associação significativa do IDATE quanto à classificação ansiedade-traço com a classificação da ansiedade pela BAI $(p=0,258)$. Contudo, quando se relacionou o BAI com a classificação ansiedade-estado do IDATE, houve associação significativa $(p=0,020)$. Todos os participantes com grau, no mínimo, leve de ansiedade pela BAI, classificaram-se como tendendo à ansiedade pelo IDATE.

Quanto à sobrecarga, os achados do ZCBI apontaram que: 20 cuidadores $(52,6 \%)$ apresentavam sobrecarga moderada; 13 (34,2\%) apresentaram ausência ou pouca sobrecarga; e $5(13,2 \%)$, sobrecarga moderada a severa. Considerando os escores de QV medidos através do WHOQOL-BREF, o maior prejuízo dos cuidadores pareceu se manifestar no domínio ambiente (percepção que o indivíduo possui sobre aspectos diversos relacionados ao ambiente em que vive), conforme informações da Tabela III.

Tabela III - Escores de qualidade de vida dos cuidadores conforme os domínios avaliados (WHOQOL-BREF). Porto Alegre, Rio Grande do Sul, 2014.

\begin{tabular}{cccc}
\hline Domínios & Média/DP & Mediana & Min-Max \\
\hline Físico & $65,7 / 19,3$ & 71,4 & $21,4-100$ \\
Psicológico & $65,7 / 20,5$ & 70,8 & $8,3-100$ \\
Social & $66,4 / 22,0$ & 66,7 & $25-100$ \\
Ambiente & $57,8 / 18,2$ & 57,8 & $21,9-100$ \\
Geral & $61,8 / 20,9$ & 62,5 & $0-100$ \\
\hline
\end{tabular}

DP: desvio padrão; Min: mínimo; Max: máximo

Quanto às associações das variáveis investigadas (idade, IDATE, BAI, BDI e ZCBI) com os domínios de QV do WHOQOL-BREF, utilizou-se o coeficiente de correlação de Pearson ou Spearman conforme o tipo de variável. Não houve associação significativa da idade do cuidador com os escores de QV, mas houve associação significativa inversa entre os escores do BDI, do BAI, do ZCBI e do IDATE-Estado e os escores de QV (exceto o domínio social). Portanto, quanto maiores os níveis de ansiedade, de depressão e de sobrecarga dos cuidadores, menores os escores de QV.

Em relação ao desenvolvimento motor da criança (GMFSC), encontraram-se as associações estatisticamente significativas somente com o domínio social da $Q V\left(r_{s}=0,355 ; p=0,029\right)$, ou seja, quanto maior o nível de desenvolvimento motor da criança (maior escore na GMFCS), maior o escore de QV do cuidador nesse domínio. A Tabela IV apresenta a associação das variáveis com os domínios de QV do WHOQOL-BREF através dos coeficientes de correlação de Pearson ou Spearman.

Tabela IV - Associação entre variáveis emocionais, qualidade de vida e desenvolvimento motor. Porto Alegre, Rio Grande do Sul, 2014.

\begin{tabular}{|c|c|c|c|c|c|c|}
\hline \multirow[b]{2}{*}{ Variáveis } & \multicolumn{5}{|c|}{ WHOQOL-BREF } & \multirow{2}{*}{$\begin{array}{c}\text { GMFCS } \\
\mathbf{r}_{\mathbf{s}}\end{array}$} \\
\hline & Físico\#\# & Psicológico & Social & Ambiente & Geral & \\
\hline IDATE - Parte I & 0,186 & 0,121 & 0,258 & 0,240 & 0,117 & 0,076 \\
\hline IDATE - Parte II & $-0,499^{* *}$ & $-0,526^{* *}$ & $-0,280$ & $-0,364^{*}$ & $-0,556^{\star \star *}$ & $-0,039$ \\
\hline BAl\# & $-0,743^{\star * *}$ & $-0,851^{* \star *}$ & $-0,657^{* * *}$ & $-0,739^{* * *}$ & $-0,759^{* * *}$ & $-0,112$ \\
\hline BDI\# & $-0,761^{\star \star * *}$ & $-0,762^{* * *}$ & $-0,726^{* * *}$ & $-0,794^{* * *}$ & $-0,737^{\star \star \star}$ & $-0,081$ \\
\hline Sobrecarga & $-0,668^{\star * *}$ & $-0,629^{* * *}$ & $-0,622^{* \star *}$ & $-0,608^{* * *}$ & $-0,664^{* * *}$ & $-0,070$ \\
\hline
\end{tabular}

GMFCS: Gross Motor Function Classification System; IDATE: Inventário de Ansiedade Traço-Estado; BDI: Beck Depression Inventory; BAl: Beck Anxiety Inventory; $\mathrm{r}_{\mathrm{s}}$ :correlação de Spearman; \#: coeficiente de correlação de Spearman; \#\#: coeficiente de correlação de Pearson; ${ }^{* * *}: p<0,001 ;{ }^{* *}: p<0,01 ;{ }^{*}: p<0,05$ 
A avaliação das médias dos escores de QV de todos os domínios conforme o nível de desenvolvimento motor, através da GMFCS, está apresentada na Figura 1. As colunas representam médias com linhas de desvio padrão. Encontrou-se associação no domínio social $(p=0,029)$.

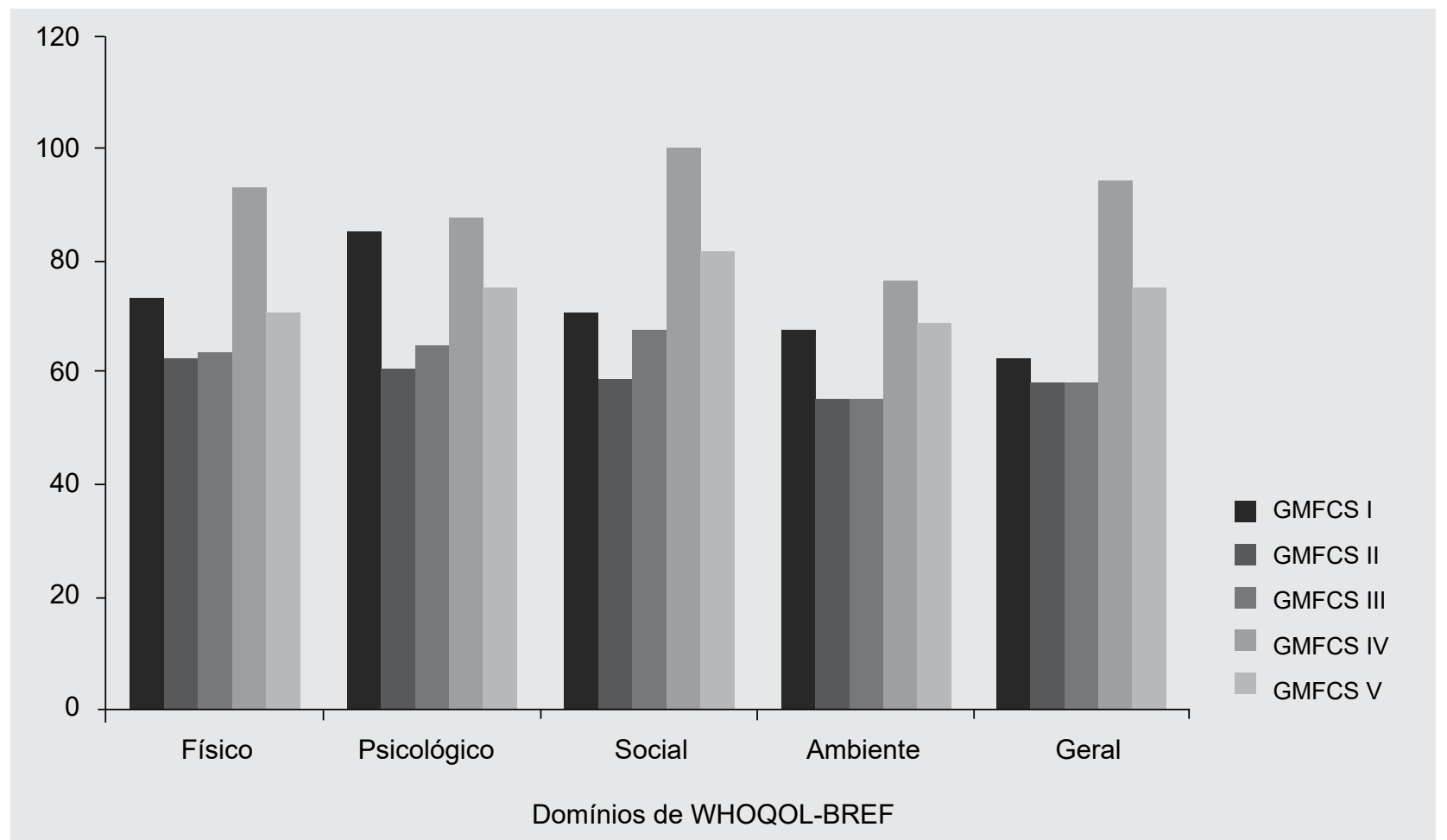

GMFCS I: Gross Motor Function Classification System nível I; GMFCS II: Gross Motor Function Classification System nível II; GMFCS III: Gross Motor Function Classification System nível III; GMFCS IV: Gross Motor Function Classification System nível IV; GMFCS V: Gross Motor Function Classification System nível V.

Figura 1 - Escores de qualidade de vida (QV) dos cuidadores conforme os domínios do WHOQOL-BREF e nível de desenvolvimento motor da criança (escala Gross Motor Function Classification System - GMFCS). Porto Alegre, Rio Grande do Sul, 2014.

Em relação à avaliação de fatores independentemente associados aos escores de QV: permaneceram associados com todos os escores de QV, o nível de sobrecarga do cuidador e os níveis de intensidade de sintomas depressivos. Nos domínios social e geral, além dessas variáveis, o nível de desenvolvimento motor da criança e a intensidade de sintomas ansiosos se associaram, independentemente, com os escores de QV. Assim, quanto maior o nível de desenvolvimento motor do paciente, melhor a QV do cuidador nesses itens. Por fim, no domínio psicológico, permaneceram associadas, além das variáveis já citadas, os níveis de intensidade dos sintomas ansiosos.

Houve associação positiva significativa entre a ZCBI (sobrecarga do cuidador) e os escores de IDATE (Estado) $\left(r_{s}=0,540 ; p<0,001\right)$, ou seja, cuidadores que relatam maior sobrecarga tendem também a apresentar maior nível de depressão. Não se encontrou tais associações com o IDATE (Traço) $\left(r_{s}=-0,072 ; p=0,666\right)$. Por fim, houve associação significativa entre o escore de sobrecarga do cuidador e a intensidade de sintomas depressivos, avaliada pelo BDI $\left(r_{s}=0,580 ; p<0,001\right)$, e de sintomas ansiosos, avaliada pelo BAl $\left(r_{s}=0,656 ; p<0,001\right)$, assim demonstrando que níveis mais elevados de depressão e ansiedade são observados em cuidadores com escores mais altos de sobrecarga.

\section{DISCUSSÃO}

Os resultados do presente estudo acompanham estudos que indicam relação entre outras condições patológicas infantis, qualidade de vida e promoção de saúde dos cuidadores. Existe concordância quanto à idade e ao sexo da amostra de cuidadores quando comparados a outros estudos, predominando cuidadores do sexo feminino como acompanhantes no processo de reabilitação das crianças $^{(35,36)}$.

A elevada proporção de mães como cuidadoras também confirma os achados apontados na literatura, e patologias como asma e câncer infantil( ${ }^{(35,36)}$, seguidas por pais e avós. Esses resultados sugerem a influência de fatores culturais e sociais, em que se observa o dever específico da mulher de exercer o papel de cuidadora da prole ${ }^{(36)}$. 
A não existência de associação significativa entre o nível de desenvolvimento motor das crianças com PC e os níveis de ansiedade, de depressão e de sobrecarga de seus cuidadores contrariou as hipóteses iniciais do presente estudo. Esse resultado poderia ser esperado, tendo em vista ocorrências similares na literatura ${ }^{(37)}$. A melhora nesse aspecto não influenciou as mudanças ocorridas na ansiedade, na depressão e na sobrecarga das mães.

No presente estudo, encontrou-se um dado significativo que compreende a existência de uma associação entre os escores da criança na GMFCS com os escores de QV dos cuidadores no domínio social. O dado possibilita discorrer a respeito de estratégias de promoção da saúde voltadas tanto para o cuidador quanto para a criança com PC, uma vez que as melhoras do desempenho motor estão relacionadas à qualidade de vida de cuidadores no domínio social ${ }^{(4,5)}$.

Identificou-se, no atual estudo, que quanto maior o grau de dificuldade motora, representados pelos escores na GMFCS, menores os índices de qualidade de vida do cuidador no âmbito social. Nesse sentido, as ações de promoção e prevenção da saúde devem estar associadas ao contexto da criança com PC, uma vez que cuidadores são afetados no domínio social devido ao agravo e à debilidade física da criança. Portanto, ressalta-se a importância da qualidade de vida do cuidador na melhoria do quadro da criança, já que é o cuidador que estará acompanhando a criança com PC em suas necessidades ${ }^{(4,5)}$.

Os resultados encontrados na presente pesquisa no domínio relacionado às relações pessoais, ao suporte e/ ou apoio, à atividade sexual e ao envolvimento de outras pessoas para auxiliar nos cuidados prestados à criança podem induzir efeitos positivos no desenvolvimento motor da criança e, consequentemente, melhora na QV do cuidador. Por outro lado, as crianças com PC e menor comprometimento motor induzem menores prejuízos às relações sociais de seus cuidadores em termos de preconceito, de exclusão social, de dedicação exclusiva ao cuidado da criança, entre outros ${ }^{(38)}$.

Outro achado do presente estudo diz respeito à percepção dos cuidadores sobre sua condição física, avaliada pela escala WHOQOL-BREF, em que se constatou uma perspectiva positiva sobre seus aspectos físicos. O achado vai ao encontro de resultados apontados em outro estudo ${ }^{(39)}$ realizado com cuidadores de crianças com neoplasia cerebral.

O domínio psicológico, terceiro melhor domínio avaliado neste estudo, considera a compreensão da percepção do indivíduo sobre a sua condição afetiva e cognitiva. Já no segundo domínio mais bem avaliado pelos cuidadores, o domínio social, encontrou-se uma grande oscilação, sendo muito bem avaliado por 15 cuidadores e muito mal pelos outros 15. Por fim, no domínio ambiental, que compreende a percepção sobre aspectos diversos relacionados ao ambiente, apareceram as piores avaliações ${ }^{(38)}$.

Percebe-se uma concordância em relação ao domínio ambiental, havendo demasiado prejuízo na avaliação da QV, o que salienta a percepção pessoal sobre o ambiente em que o cuidador vive. Isto indica os vários aspectos envolvidos na QV como estrutura de moradia, de lazer e outros indicadores ${ }^{(38)}$.

Observou-se ainda, no presente estudo, a ausência de sobrecarga sobre 13 cuidadores, resultado similar aos obtidos em outro estudo ${ }^{(39)}$. Em alguns casos, fatores como problemas comportamentais da criança, particularmente as interações sociais, a sua dificuldade de inclusão escolar ou as condições econômicas desfavoráveis podem ser preditivos da sobrecarga do cuidador. Esse achado pode indicar que, mesmo que existam fatores preditivos para sobrecarga do cuidador, nem sempre esses aspectos devem ser considerados como fatores exclusivos para que a sobrecarga do cuidador torne-se presente ${ }^{(39)}$.

Apesar de não ter sido confirmada a expectativa inicial de que haveria associação entre QV dos cuidadores e o nível de desenvolvimento motor das crianças, os resultados indicam a importância de estabelecer atenção para o desenvolvimento de estratégias de promoção à saúde e prevenção direcionadas para as necessidades dos cuidadores de crianças com PC ${ }^{(22)}$.

\section{CONCLUSÃO}

Conclui-se que a interação entre a qualidade de vida e o estado emocional dos cuidadores não estão relacionados ao nível de desenvolvimento motor das crianças com paralisia cerebral. Contudo, é importante salientar que o aumento nos níveis de ansiedade, de depressão e de sobrecarga correlaciona-se com a diminuição dos escores de QV dos cuidadores investigados. 


\section{CONTRIBUIÇÕES}

Jandara de Moura Souza contribuiu na elaboração e no delineamento do estudo; na aquisição, análise e interpretação de dados; na redação e/ou revisão do manuscrito. Fabiana Rita Câmara Machado e Priscilla Pereira Antunes contribuíram na aquisição, na análise e na interpretação de dados; e na redação do manuscrito. Antônio Cardoso dos Santos contribuiu na elaboração e no delineamento do estudo; e na revisão do manuscrito. Daniela Centenaro Levandoswki contribuiu na elaboração e no delineamento do estudo; na análise e interpretação de dados; e na revisão do manuscrito. Alcyr Alves de Oliveira Junior contribuiu na elaboração e no delineamento do estudo, na análise e interpretação de dados; e na redação e/ou revisão do manuscrito.

\section{REFERÊNCIAS}

1. Houtrow A, Kang T, Newcomer R. In-home supportive services for individuals with cerebral palsy in California [Internet]. J Pediatr Rehabil Med. 2012 [acesso em 2018 Fev 12];5(3):187-95. Disponível em: https://www.ncbi. nlm.nih.gov/pmc/articles/PMC3610921/.doi:10.3233/PRM-2012-0211

2. Ryan JM, Allen E, Gormley J, Hurvitz EA, Peterson M. The risk, burden, and management of non-communicable diseases in cerebral palsy: a scoping review [Internet]. Dev Med Child Neur. 2018 [acesso em 2018 Ago 20];60(8):753-764. Disponível em: https://www.ncbi.nlm.nih.gov/pubmed/29572812.doi10.1111/dmcn.13737

3. Morbeck TNO, Pereira LM, Assis-Madeira EA. Dependência de crianças com paralisia cerebral e qualidade de vida de suas mães [Internet]. Cad de Pós-Graduação em Distúrbios do Desenv. 2014 [acesso em $2018 \mathrm{Fev}$ 20];14(2):8-20. Disponível em: http://editorarevistas.mackenzie.br/index.php/cpgdd/article/viewFile/11272/7000. ISSN1809-4139

4. Volpini M, Brandão MB, Pereira LB, Mancini MC, Assis MG. Mobilidade sobre rodas: a percepção de pais de crianças com paralisia cerebral [Internet]. Cad Ter Ocup UFSCar. 2013 [acesso em 2018 Maio 12];21(3):471-8. Disponível em: http://www.cadernosdeto.ufscar.br/index.php/cadernos/article/viewFile/907/459ISSN0104-4931

5. Machado FRC, Antunes PP, Souza JM, Santos AC, Levandowski DC, Oliveira AA. Motor improvement using motion sensing game devices for cerebral palsy rehabilitation [Internet]. J Mot Behav. 2017 May-Jun [cited 2018 Abr 18];49(3):273-80. Disponível em: https://www.ncbi.nlm.nih.gov/pubmed/27593342.doi10.1080/00222895.2 016.1191422

6. Moreira RS, Magalhães LC, Alves CRL. Effect of preterm birth on motor development, behavior, and school performance of school-age children: a systematic review [Internet]. J Pediatr. 2014 [acesso em 2018 Abr 24];90(2):11934. Disponível em: http://www.scielo.br/scielo.php?script=sci_arttext\&pid=S0021-75572014000200119\&lng=en \&nrm=iso.ISSN1678-4782

7. Silva M, Daltrário S. Paralisia cerebral: desempenho funcional após treinamento da marcha em esteira. Fisioter Mov [Internet]. 2017 [acesso em 2018 Mar 17];21(3):109-15. Disponível em: https://periodicos.pucpr.br/index. php/fisio/article/view/19179

8. Camargos ACR, Lacerda TTB, Barros TV, Silva GC, Parreiras JT, Vidal THJ. Relação entre independência funcional e qualidade de vida na paralisia cerebral. Fisioter Mov [Internet]. 2012 [acesso em 2018 Mar 15];25(1):83-92. Disponível em: http://www.scielo.br/pdf/fm/v25n1/a09v25n1

9. Knox V, Evans AL. Evaluation of the functional effects of a course of Bobath therapy in children with cerebral palsy: a preliminary study. Dev Med Child Neurol [Internet]. 2002 [acesso em 2018 Abr 21];44(7):447-60. Disponível em: https://www.cambridge.org/core/journals/developmental-medicine-and-child-neurology/article/evaluation-ofthe-functional-effects-of-a-course-of-bobath-therapy-in-children-with-cerebral-palsy-a-preliminary-study/F9DC5 D8828D12A272A959019678E3364\#fndtn-information

10. Santos TL, Quaresma MP. Aplicações de toxina botulíica tipo A como um meio terapêutico em doenças distônicas. Revinter [Internet]. 2018 [acesso em 2018 Jul 20];11(1):84-99. Disponível em: http://www.revistarevinter.com. br/autores/index.php/toxicologia/article/view/352

11. Rosa ACA, Matos MR. Classificação funcional de indivíduos com paralisia cerebral de acordo com a escala de avaliação GMFCS. Rev Univap [Internet]. 2017 [acesso em 2018 Abr 21];22(40):1-4. Disponível em: https:// revista.univap.br/index.php/revistaunivap/article/view/861 
12. Santos JS. Aspectos motores, de comunicação, sono-vigília e melatonina na paralisia cerebral [dissertação]. São Paulo: Universidade Estadual Paulista; 2017.

13. Cruz MAA, Passos ATP, Fernandes MFG. Perfil funcional de crianças com paralisia cerebral atendidas no setor multidisciplinar do núcleo de tratamento e estimulação precoce (programa de intervenção precoce no desenvolvimento na primeira infância-QH.2011.PG.0861). Rev Encontros Univ UFC [Internet]. 2017 [acesso em 2018 Jun 21];2(1):3926. Disponível em: http://www.periodicos.ufc.br/eu/article/view/28950/64154

14. Freitag VL, Milbrath VM, Schneider FVM, Lange C. O impacto do diagnóstico de paralisia cerebral para a família: revisão integrativa. J Nurs Health [Internet]. 2017 [acesso em 2018 Abr 10];7(1):89-100. Disponível em: https:// periodicos.ufpel.edu.br/ojs2/index.php/enfermagem/article/view/6064.ISSN2236-1987

15. Oliveira AKC, Matsukurab TS. Estresse e apoio social em cuidadores de crianças com paralisia cerebral. Cad Ter Ocup UFSCar. 2013;21(3):493-503.

16. Almeida KM, Fonseca, BM, Gomes AA, Oliveira MX. Fatores que influenciam a qualidade de vida de cuidadores de paralisados cerebrais. Fisioter Mov [Internet]. 2013 [acesso em 2018 Jul 18];26(2):307-14. Disponível em: http://www.scielo.br/scielo.php?pid=S0103-51502013000200007\&script=sci_abstract\&tlng=pt

17. Freitag VL, Milbrath VM, Motta MGC. Mãe-cuidadora de criança/adolescente com Paralisia Cerebral: o cuidar de si. Enferm Glob [Internet]. 2018 [acesso em 2018 Abr 21];17(50):337-48. Disponível em: http://scielo.isciii. es/pdf/eg/v17n50/pt_1695-6141-eg-17-50-325.pdf.ISSN1695-6141

18. Simões CC, Silva LS, Santos MR, Misko MD, Bousso RS. A experiência dos pais no cuidado dos filhos com paralisia cerebral. Rev Eletrônica Enferm [Internet]. 2013 [acesso em 2017 Dez 15];15(1):138-45. Disponível em: https://www.revistas.ufg.br/fen/article/view/13464/15531

19. Silva SSC, Pontes FAR. Rotina de famílias de crianças com paralisia cerebral. Educ Rev [Internet]. 2016 [acesso em 2018 Abr 20];59:65-78. Disponível em: http://www.scielo.br/scielo.php?script=sci_arttext\&pid=S010440602016000100065\&lng=en\&nrm=isoaccess

20. Coimbra CS, Müller AB. Comprometimento funcional, condição socioeconômica e sobrecarga de cuidado na paralisia cerebral. Rev Bras Qual Vida. 2017;9(3):208-22.

21. Silva RS, Fedosse E. Perfil sociodemográfico e qualidade de vida de cuidadores de pessoas com deficiência intelectual. Cad Bras Ter Ocup. 2018;26(2):357-66.

22. Fleck MPA. O instrumento de avaliação de qualidade de vida da Organização Mundial da Saúde (WHOQOL-100): características e perspectivas. Ciênc Saúde Coletiva [Internet]. 2000 [acesso em 2018 Jun 14];5(1):33-8. Disponível em: http://www.redalyc.org/articulo.oa?id=63050104.ISSN1413-8123

23. Minayo MCS, Hartz ZMA, Buss PM. Qualidade de vida e saúde: um debate necessário. Ciênc Saúde Coletiva [Internet]. 2000 [acesso em 2018 Jun 12];5(1):23-46. Disponível em: http://www.redalyc.org/articulo.oa?id=63050102

24. Miura RT, Petean EB. Paralisia cerebral grave: o impacto na qualidade de vida de mães cuidadoras. Mudanças [Internet]. 2012 [acesso em 2018 Jul 10];20(1-2):7-12. Disponível em: https://www.metodista.br/revistas/revistasims/index.php/MUD/article/view/3146/3126

25. Falchetti ACB, Fernandes JB, Elias A. Percepção de familiares frente à acessibilidade de reabilitação para crianças com paralisia cerebral. Rev Baiana Saúde Pública [Internet]. 2013 [acesso em 2018 Jul 08];37(2):32234. Disponível em: http://files.bvs.br/upload/S/0100-0233/2013/v37n2/a4417.pdf

26. Morreti GS, Moreira KA, Pereira SR. Qualidade de vida dos cuidadores de crianças com paralisia cerebral da Amazônia sul ocidental [Internet]. Rev Bras Promoç Saúde. 2012 [acesso em 2018 Abr 12];25(1):30-6. Disponível em: http://periodicos.unifor.br/RBPS/article/view/2207/2408.ISSN1806-1230

27. Seidl EMF, Zannon CMLC. Qualidade de vida e saúde: aspectos conceituais e metodológicos. Cad Saúde Pública [Internet] 2004 [acesso em 2017 Dez 12];20(2): 580-88. Disponível em: http://www.scielo.br/pdf/csp/v20n2/27. pdf

28. Cunha J. Manual da versão em português das escalas de Beck. São Paulo: Casa do Psicólogo; 2001.

29. Beck AT, Steer RA, Ball R, Ranier W. Comparison of beck depression inventories -IA and -II in psychiatric outpatients. J Pers Assess. 1996;67(3):588-97. 
30. Fioravanti ACM, Santos LF, Maissonette S, Cruz APM, Landeira-Fernandez J. Avaliação da estrutura fatorial da Escala de Ansiedade-Traço do IDATE [Internet]. Aval Psicol [Internet] 2006 [acesso em 2018 Jun 10];5(2):21724. Disponível em: https://dialnet.unirioja.es/servlet/articulo?codigo $=5115194$

31. Ferreira MC, Naccio BLD, Otsuka MYC, Barbosa AM, Corrêa PFL, Gardenghi G. Avaliação do índice de sobrecarga de cuidadores primários de crianças com paralisia cerebral e sua relação com a qualidade de vida e aspectos socioeconômicos. Acta Fisiátrica [Internet]. 2015 [acesso em 2018 Jun 17];22(1):9-13. Disponível em: http:// www.revistas.usp.br/actafisiatrica/article/view/103894

32. Zarit SH, Reever SH, Back-Peterson JKE. Relatives of the impaired elderly: correlates of the feelings of burden. Gerontologist [Internet]. 1980 [acesso em 2018 Maio 25];20(6):649-55. Disponível em: https://doi.org/10.1093/ geront/20.6.649

33. Oliveira A, Golin M, Cunha M. Aplicabilidade do Sistema de Classificação da Função Motora Grossa (GMFCS) na paralisia cerebral: revisão da literatura. Arq Bras Ciênc Saúde. 2010;35(3):220-4.

34. Silva LM, Monteiro ES, Paiva SSC, Torres MV, Carvalho MEIM. Efeitos da equoterapia na função motora grossa de pacientes com encefalopatia crônica não progressiva. Rev Neurociênc [Internet]. 2015 [acesso em $2018 \mathrm{Abr}$ 20];23(1):16-22. Disponível em: http://www.revistaneurociencias.com.br/edicoes/2015/2301/original/941original. pdf

35. Braccialli LMP, Ferronato BP, Rubira ABFA, Reganhan WG, Sankako AN, Gonçalves AG, et al. Qualidade de vida de crianças com paralisia cerebral: percepção de cuidadores [Internet]. Rev Bras Qual Vida. 2016 [acesso em 2018 Fev 18];8(1):57-69. Disponível em: https://periodicos.utfpr.edu.br/rbqv/article/view/3700/2593

36. Fernandes ICF. Qualidade de vida de cuidadores de crianças e adolescentes asmáticos [dissertation]. Goiânia: Universidade Federal de Goiás; 2014.

37. Ones K, Yilmaz E, Cetinkaya B, Caglar N. Assessment of the quality of life of mothers of children with cerebral palsy (primary caregivers) [Internet]. Neurorehabil Neural Repair. 2005 [acesso em 2018 Jun 2019];19(3):232-7. Disponível em: https://doi.org/10.1177/1545968305278857

38. Figueiredo AA. Avaliação da qualidade de vida de cuidadores de pacientes pediátricos com paralisia cerebral tetraparética [dissertation]. Campinas: Universidade Estadual de Campinas; 2017.

39. Mugno D, Ruta L, D'Arrigo VG, Mazzone L. Impairment of quality of life in parents of children and adolescents with pervasive developmental disorder. Health Qual Life Outcomes. 2007;7(27):1-9.

\section{Endereço do primeiro autor:}

Jandara de Moura Souza

Universidade Federal de Ciências da Saúde de Porto Alegre - UFCSPA

Rua Sarmento Leite, 245

Bairro: Centro Histórico

CEP: 90050-170 - Porto Alegre - RS - Brasil

E-mail: jandara.souza@gmail.com

\section{Endereço para correspondência:}

Alcyr Alves de Oliveira Júnior

Universidade Federal de Ciências da Saúde de Porto Alegre - UFCSPA

Rua Sarmento Leite, 245

Bairro: Centro Histórico

CEP: 90050-170 - Porto Alegre - RS - Brasil

E-mail: alcyr.oliveirajr@gmail.com 